\title{
Discovery of novel anti-HIV active G-quadruplex-forming oligonucleotides $\dagger \ddagger$
}

\author{
Giovanni Di Fabio, ${ }^{* a}$ Jennifer D'Onofrio, ${ }^{a}$ Mariateresa Chiapparelli, ${ }^{a}$ Bart Hoorelbeke, ${ }^{b}$ \\ Daniela Montesarchio, ${ }^{a}$ Jan Balzarini ${ }^{b}$ and Lorenzo De Napoli ${ }^{a}$
}

Received 2nd November 2010, Accepted 23rd November 2010

DOI: $10.1039 / \operatorname{cocc04751a}$

\begin{abstract}
A series of $\left.\mathrm{d}^{\mathbf{5}^{\prime}} \mathrm{TGGGAG}^{\mathbf{3}^{\prime}}\right)$ sequences, $5^{\prime}$-conjugated with a variety of aromatic groups through phosphodiester linkages, were synthesized, showing CD spectra diagnostic of parallelstranded, tetramolecular G-quadruplex structures. When tested for anti-HIV-1 and HIV-2 activity, potent inhibition of HIV-1 infection in CEM cell cultures was found, associated with high selectivity index values. Surface Plasmon Resonance assays revealed specific binding to HIV-1 gp120 and gp41.
\end{abstract}

Oligonucleotide-based aptamers ${ }^{1}$ frequently contain peculiar secondary structures, such as hairpin or G-quadruplex motifs. Among these, G-quadruplexes ${ }^{2}$ are particularly interesting. In fact, several G-rich synthetic oligodeoxyribonucleotides (ODNs) have shown promising biological properties, ranging from anticancer to anti-HIV activities; in all cases, G-quadruplex formation was found to be a crucial prerequisite in determining these biological effects. $^{3}$

A number of G-quadruplex-forming oligonucleotides have been developed as potential anti-HIV drugs. ${ }^{4}$ To explain the antiviral activity of G-rich oligomers two mechanisms have been proposed: inhibition of viral entry into the cell, with the V3 loop of the gp120 envelope protein indicated as the primary target, and/or inhibition of HIV-1 integrase. ${ }^{5}$

In the search for ODNs endowed with relevant antiviral properties, Hotoda and his group investigated a series of G-quadruplex-forming ODNs, finally focusing on modified $\mathrm{d}\left({ }^{5^{\prime}} \mathrm{TGGGAG}^{3^{\prime}}\right)$ ODNs. Several d $\left(^{5^{\prime}} \mathrm{TGGGAG}^{3^{\prime}}\right)$ oligomers, conjugated with different groups at the $5^{\prime}$ or at the $3^{\prime}$-end, connected through an ether bond, were synthesized and tested for anti-HIV-1 activity. While unmodified $\mathrm{d}\left({ }^{5^{\prime}} \mathrm{TGGGAG}^{3^{\prime}}\right)$ had no antiviral activity, the oligomer bearing the 3,4-di(benzyloxy)benzyl group at the $5^{\prime}$-end (DBB-TGGGAG) strongly inhibited HIV-1 ( $\mathrm{IC}_{50}=0.37 \mu \mathrm{M}$ on MT-4 cells $)$

\footnotetext{
${ }^{a}$ Dipartimento di Chimica Organica e Biochimica,

Università degli Studi di Napoli "Federico II",

Complesso Universitario di Monte S. Angelo, via Cintia, 4,

I-80126 Napoli, Italy.E-mail: giovanni.difabio@unina.it;

Fax: + 39 081-674393; Tel: + 39 081-674001

${ }^{b}$ Rega Institute for Medical Research, Katholieke Universiteit Leuven, 10 Minderbroedersstraat, B-3000 Leuven, Belgium

$\dagger$ Abbreviations: $\mathrm{CE}=2$-cyanoethyl; $\mathrm{CPG}=$ Controlled Pore Glass; DBB $=3$,4-dibenzyloxybenzyl; DCM = dichloromethane; DIEA = $N, N$-diisopropylethylamine; DMT $=4,4^{\prime}$-dimethoxytriphenylmethyl; ipropyl $=$ isopropyl; ODN $=$ oligodeoxyribonucleotide; $p=$ phosphodiester linkage.

$\ddagger$ Electronic supplementary information (ESI) available: Experimental methods, synthetic procedures and characterization of phosphoramidites 1-5. CD spectra, CD-monitored melting curves, SPR-plots and anti-HIV-1 RT activity for ODNs $\mathbf{I}-\mathbf{V}$ are also available. See DOI: $10.1039 / \mathrm{c} 0 \mathrm{cc} 04751 \mathrm{a}$
}

without showing cytotoxicity up to $40 \mu \mathrm{M} .{ }^{6}$ Studies on selected analogues of this sequence indicated that G-quadruplex formation, as well as the presence of large aromatic substituents at the $5^{\prime}$-end, are essential for the anti-HIV activity, attributed to the interaction of the $5^{\prime}$-substituted oligomers with the V3 loop of gp120 as well as with the CD4 binding site in the viral gp120. For a better insight into the structure-activity relationships of these G-rich ODNs, recently we analyzed, using an integrated approach combining CD, DSC and molecular modeling studies, some representative anti-HIV active Hotoda's 6-mers, in comparison with unmodified $\mathrm{d}\left({ }^{5^{\prime}} \mathrm{TGGGAG}^{3^{\prime}}\right)$. On the basis of the obtained results, we concluded that the aromatic groups at the 5 '-end play a major role on the G-quadruplex stability, dramatically enhancing both the rate of formation and stability of the quadruplex complexes. ${ }^{7}$

The insertion of $5^{\prime}$-modifications through ether bonds requires the dedicated, sometimes cumbersome synthesis of a specific $5^{\prime}$-modified nucleoside $3^{\prime}$-phosphoramidite building block for each desired conjugation. ${ }^{6,7}$

Aiming at new and more effective anti-HIV agents using $\mathrm{d}\left({ }^{5^{\prime}}\right.$ TGGGAG $\left.^{3^{\prime}}\right)$ as a lead sequence, a general strategy based on an on-line fully automated synthesis of modified ODNs, requiring only straightforward and efficient manipulations of the conjugating agent, would be highly desirable. With the final goal to expand the repertoire of accessible end-modified G-rich ODNs, we reasoned that linking reporter groups to the ODN chain through phosphodiester bonds results into a simpler and more general synthetic route. ${ }^{8}$

We here describe the design and characterization of a mini-library of $\left.\mathrm{d}^{5^{\prime}} \mathrm{TGGGAG}^{3^{\prime}}\right)$ ODNs carrying aryl groups at the $5^{\prime}$-end connected through phosphodiester bonds, and the evaluation of these compounds as anti-HIV agents.

For this purpose we initially converted a set of commercially available phenols and aromatic alcohols into the corresponding phosphoramidite derivatives by reaction with 2-cyanoethyl$\mathrm{N}, \mathrm{N}$-diisopropylaminochlorophosphoramidite and DIEA in anhydrous DCM. The identity of compounds 1-5 (Fig. 1), all obtained in good yields ( $\geq 85 \%$ ), was confirmed by NMR $\left({ }^{1} \mathrm{H}\right.$ and $\left.{ }^{31} \mathrm{P}\right)$ and MS data. $f$

Sequences $\mathbf{I}-\mathbf{V}$ were synthesized starting from a CPG-dG support (Scheme 1), exploiting commercially available $3^{\prime}$-phosphoramidite-2'-deoxyribonucleoside monomers in a standard solid phase $\beta$-cyanoethyl phosphoramidite chemistry. The modification at the $5^{\prime}$-end was introduced using, respectively, phosphoramidites 1-5 in the last coupling step of the ODN chain assembly. The resulting supports were reacted with $\mathrm{Et}_{3} \mathrm{~N} /$ pyridine $(1: 1, \mathrm{v} / \mathrm{v})$, at $50{ }^{\circ} \mathrm{C}$ for $1 \mathrm{~h}$, followed by treatment with conc. aq. ammonia at $50{ }^{\circ} \mathrm{C}$ for $5 \mathrm{~h}$, allowing 

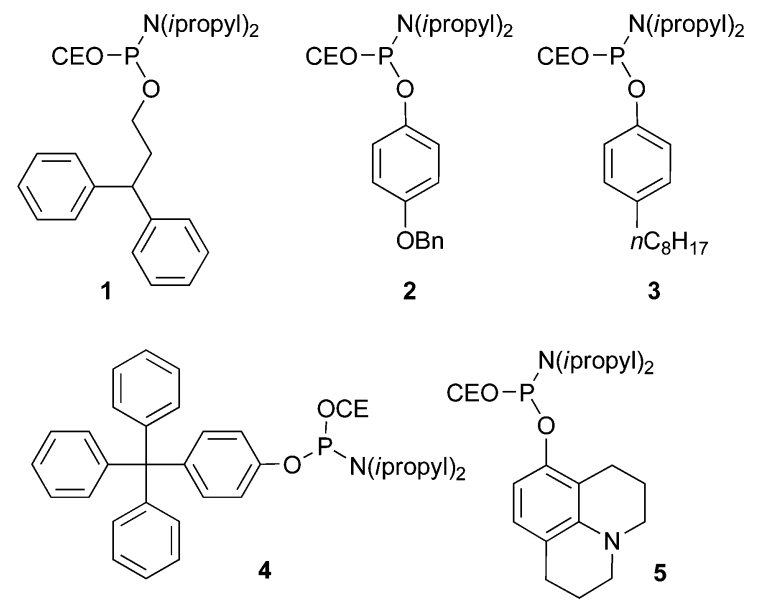

Fig. 1 Set of the here investigated aromatic phosphoramidite labels.

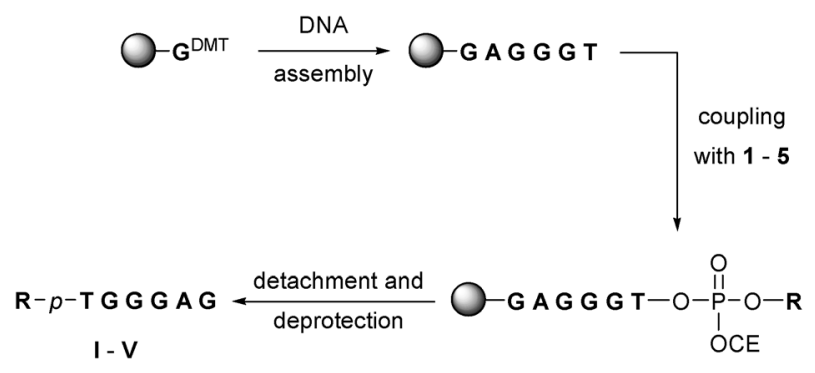

Scheme 1 Synthetic scheme for the preparation of ODNs I-V.

full deprotection and detachment from the solid support of target oligomers $\mathbf{I}-\mathbf{V}$.

The combined filtrates and washings were concentrated in vacuo, redissolved in $\mathrm{H}_{2} \mathrm{O}$, analyzed and purified by HPLC. $\ddagger$

The modified oligonucleotides were characterized by MALDI-TOF mass spectrometry, in all cases giving masses in accordance with the expected values (Table 1). In a typical experiment, starting from $100 \mathrm{mg}$ of CPG-dG support, 80-100 OD units of purified $\mathbf{I}-\mathbf{V}$ were obtained.

In order to study the influence of the aromatic groups at the $5^{\prime}$-end of the oligonucleotide chains on their ability to form quadruplex structures, a CD analysis was undertaken

Table 1 Oligonucleotide MS characterization and melting temperatures of the quadruplexes formed by ODNs I-V

\begin{tabular}{|c|c|c|c|}
\hline \multirow[b]{2}{*}{ Sequences } & \multicolumn{2}{|l|}{ MS data } & \multirow[b]{2}{*}{$\mathrm{Tm} /{ }^{\circ} \mathrm{C}$} \\
\hline & Calcd $[\mathrm{M}]$ & Found $(m / z)$ & \\
\hline TGGGAG & 1871.36 & $1872.01[\mathrm{MH}]^{+a}$ & $41 \pm 1^{a}$ \\
\hline I & 2145.43 & $\begin{array}{l}2146.13[\mathrm{MH}]^{+} \\
2171.70[\mathrm{MNa}]^{+} \\
2184.77[\mathrm{MK}]^{+}\end{array}$ & $85.0 \pm 0.5$ \\
\hline II & 2133.39 & $\begin{array}{l}2134.05[\mathrm{MH}]^{+} \\
2155.75[\mathrm{MNa}]^{+}\end{array}$ & $83.5 \pm 0.5$ \\
\hline III & 2139.48 & $\begin{array}{l}2143.21[\mathrm{MH}]^{+} \\
2165.02[\mathrm{MNa}]^{+} \\
2183.19[\mathrm{MK}]^{+}\end{array}$ & $85.0 \pm 0.5$ \\
\hline IV & 2269.46 & $\begin{array}{l}2270.33[\mathrm{MH}]^{+} \\
2293.20[\mathrm{MNa}]^{+}\end{array}$ & $59.0 \pm 0.5$ \\
\hline $\mathbf{V}$ & 2122.43 & $\begin{array}{l}2123.73[\mathrm{MH}]^{+} \\
2162.12[\mathrm{MNa}]^{+}\end{array}$ & $57.5 \pm 0.5$ \\
\hline
\end{tabular}

on oligomers $\mathbf{I}-\mathbf{V}$ in comparison with the corresponding unmodified $\mathrm{d}\left({ }^{5^{\prime}} \mathrm{TGGGAG}^{3^{\prime}}\right)$ oligomer. $\mathrm{CD}$ spectra at $20{ }^{\circ} \mathrm{C}$, for all the sequences, showed a typical positive band at $264 \mathrm{~nm}$ and a negative band at $243 \mathrm{~nm}$, diagnostic of parallel-stranded tetramolecular quadruplex structures. $\ddagger$ From the CD-monitored melting profiles, registere at $264 \mathrm{~nm}$ and $0.5^{\circ} \mathrm{C} \mathrm{min}^{-1}$ heating rate, the corresponding Tm values were obtained (Table 1). These data clearly showed that all the 6-mers formed G-quadruplexes with strongly increased thermal stability with respect to the unmodified sequence. The stabilization is less pronounced for ODNs IV and $\mathbf{V}$, carrying the bulkiest groups in the series, probably less efficient, by steric hindrance, in promoting a well-packed cluster at the 5'-end of the quadruplex structure.

When tested for anti-HIV-1 and anti-HIV-2 activity (Table 2), sequences II and III most potently inhibited HIV-1 replication in CEM cell cultures in vitro $\left(\mathrm{EC}_{50}=0.061\right.$ and $0.13 \mu \mathrm{M}$, respectively). Their selectivity indices amounted to $>1000$. Their anti-HIV-2 activity was markedly less pronounced than noticed for HIV-1. Although ODNs I and V were less active than II and III, but with anti-HIV-1 activity values comparable to Hotoda's DBB-TGGGAG $\left[\mathrm{IC}_{50}=0.37 \mu \mathrm{M}\right.$ on MT-4 cells, from ref. 6], these sequences are nonetheless very interesting for their very favourable selectivity indices (S.I $>3 \times 10^{2}$ ).

Compounds $\mathbf{I}$ to $\mathbf{V}$ were found to be poorly inhibitory against recombinant HIV-1 reverse transcriptase. $\ddagger$ Compounds II, IV and $\mathbf{V}$, showing different antiviral potencies, were also investigated by SPR analysis for their direct interaction with HIV-1 gp120 and gp41. The three compounds specifically and dosedependently bind to both gp120 and gp41. In these experiments, low-density gp120 (822 RU)- and gp41 (637 RU)-bound chips were used with relatively high concentrations of the tested compounds (40 to $160 \mu \mathrm{M}$ ). The $K_{\mathrm{D}}$ affinity constants are shown in Table 3. However, gp120 has also been bound on a chip at high density (9492 RU) and exposed to low compound concentrations (1.25 to $20 \mu \mathrm{M})$ (Table 3 ). The highest affinity for gp120 (and gp41) was recorded for compound $\mathbf{V}\left(K_{\mathrm{D}}\right.$ being $47.7 \mu \mathrm{M}$ as determined on the low-gp120 density chip or $10.3 \mu \mathrm{M}$ on the high-gp120 density chip). It should be noticed that both experimental conditions are not ideal for performing binding kinetics of these (small-size) compounds. The highgp120 density chip experiments have the advantage to have a higher binding signal sensitivity due to the abundance of potential binding interaction points (gp120 molecules). But when a compound is released from gp120, it can easily rebind to another gp120 molecule bound to the same chip. The latter phenomenon may result in an increased apparent affinity due to an apparent slower off-rate. On the contrary, when using a low gp120 density chip, rebinding is less likely to occur, but stapling of the molecules to each other at the higher compound concentrations may result in a lesser pronounced apparent affinity. However, under both experimental conditions, the $K_{\mathrm{D}}$ values were within the same order of magnitude. There is no straight correlation between the efficiency of envelope binding and the eventual antiviral activity of the test compounds. It cannot be excluded that compounds II, IV and $\mathbf{V}$ bind at different epitopes of gp120. Some of these epitopes can be neutralizing (explaining the anti-HIV activity) whereas other 
Table 2 Anti-HIV-1 and anti-HIV-2 activity and cytostatic properties of ODNs I-V in human T-lymphocyte (CEM) cells

\begin{tabular}{|c|c|c|c|c|}
\hline \multirow[b]{2}{*}{ Sequences } & \multicolumn{2}{|l|}{$\mathrm{EC}_{50}{ }^{a} / \mu \mathrm{M}$} & \multirow[b]{2}{*}{$\mathrm{CC}_{50}{ }^{b} / \mu \mathrm{M}$} & \multirow[b]{2}{*}{ S.I. ${ }^{c}$} \\
\hline & $\mathrm{EC}_{50}(\mathrm{HIV}-1)$ & $\mathrm{EC}_{50}(\mathrm{HIV}-2)$ & & \\
\hline DBB-TGGGAG & $0.37 \pm 0.06^{d}$ & - & $>40^{d}$ & $>1.1 \times 10^{2}$ \\
\hline I & $0.51 \pm 0.25$ & $26 \pm 9.1$ & $>160$ & $>3.1 \times 10^{2}$ \\
\hline II & $0.061 \pm 0.04$ & $27 \pm 6.7$ & $107 \pm 6.7$ & $1.8 \times 10^{3}$ \\
\hline III & $0.13 \pm 0.02$ & $32 \pm 0.0$ & $158 \pm 2.2$ & $1.2 \times 10^{3}$ \\
\hline IV & $4.3 \pm 0.0$ & $>32$ & $98 \pm 4.5$ & 22 \\
\hline $\mathbf{V}$ & $0.50 \pm 0.33$ & $62 \pm 4.5$ & $>160$ & $>3.2 \times 10^{2}$ \\
\hline
\end{tabular}

Table 3 Kinetic data for the interactions of test compounds with HIV gp120 and gp41, immobilized on the sensor chip $\ddagger$

\begin{tabular}{lccll}
\hline Sequences & Env. & $K_{\mathrm{D}} / \mu \mathrm{M}$ & $K_{\mathrm{a}}(1 / \mathrm{M} \mathrm{s})$ & $K_{\mathrm{d}}(1 / \mathrm{s})$ \\
\hline \multicolumn{2}{l}{ Low-gp120 and -gp41 } & density chip & & \\
$\mathbf{V}$ & gp120 & 47.7 & $1.28 \times 10^{2}$ & $6.12 \times 10^{-3}$ \\
$\mathbf{V}$ & gp41 & 15.4 & $9.22 \times 10^{2}$ & $1.42 \times 10^{-2}$ \\
II & gp120 & 205 & $5.86 \times 10^{2}$ & $1.20 \times 10^{-1}$ \\
II & gp41 & 223 & $3.82 \times 10^{2}$ & $8.52 \times 10^{-2}$ \\
IV & gp120 & 58.9 & $1.92 \times 10^{3}$ & $1.13 \times 10^{-1}$ \\
IV & gp41 & 130 & $1.69 \times 10^{2}$ & $2.21 \times 10^{-2}$ \\
High-gp120 density chip & & & \\
$\mathbf{V}$ & gp120 & 10.3 & $3.13 \times 10^{2}$ & $3.23 \times 10^{-3}$ \\
II & gp120 & 21.4 & $8.60 \times 10^{3}$ & $1.85 \times 10^{-1}$ \\
IV & gp120 & N.D. ${ }^{a}$ & N.D. & N.D. \\
${ }^{a}$ Not determined. & & & \\
\hline
\end{tabular}

epitopes may not. It has indeed been reported earlier that antibodies against Man $\alpha 1,2-$ Man $\alpha 1,2-$ Man oligosaccharides can efficiently bind to HIV-1 gp120 without neutralization of the virus in cell culture. ${ }^{9}$

In conclusion a mini-library of $\left.\mathrm{d}^{5^{\prime}} \mathrm{TGGGAG}^{3^{\prime}}\right)$ ODNs, conjugated with different aromatic groups at the $5^{\prime}$-end through a phosphodiester bond, has been synthesized and tested for anti-HIV activity. The synthetic strategy here adopted is based on the simple conversion of a set of aromatic alcohols or phenols into the corresponding phosphoramidites (1-5), incorporated in the last step of the ODN chain assembly exploiting a standard, fully automated phosphoramidite-based strategy. The target $5^{\prime}$-end conjugated-ODNs I-V were prepared and characterized by MALDI-TOF MS and CD analysis. CD spectra for all the sequences were diagnostic of parallelstranded tetramolecular G-quadruplexes. The CD-monitored melting profiles, registered at $264 \mathrm{~nm}$, indicated that the insertion of an aromatic group at the $5^{\prime}$-end confers highly improved stability with respect to the unmodified complex. The stabilization is less pronounced $\left(\Delta \mathrm{Tm}=16-18^{\circ} \mathrm{C}\right)$ for $\mathbf{I V}$ and $\mathbf{V}$, having the bulkiest groups within the studied set.

Several compounds showed pronounced activity preferentially against HIV-1(IIIB). They also showed high binding affinities for the HIV-1 envelope gp120 and gp41.

Structural characterization studies, combining NMR analysis with molecular modeling studies, are currently underway in our laboratories to get a detailed insight into the three-dimensional features of these $5^{\prime}$-modified quadruplex complexes, and a more complete picture of their structureactivity relationships.

Following the here described approach, the synthesis of a new library of $5^{\prime}$-conjugated $\mathrm{d}\left({ }^{5^{\prime}} \mathrm{TGGGAG}^{3^{\prime}}\right)$ ODNs is in progress in order to further optimize their antiviral activity.

We acknowledge MIUR (PRIN) for grants in support of this investigation. We also thank C.I.M.C.F., Università degli Studi di Napoli "Federico II", for the NMR, MS, and CD facilities. Financial support to J.B. was provided by the K. U. Leuven (GOA no. 10/014).

\section{Notes and references}

1 S. M. Nimjee, C. P. Rusconi and B. A. Sullenger, Annu. Rev. Med., 2005, 56, 555-583; C. Pestourie, B. Tavitian and F. Duconge, Biochimie, 2005, 87, 921-930.

2 J. L. Huppert, Chem. Soc. Rev., 2008, 37, 1375-1384; H. J. Lipps and D. Rhodes, Trends Cell Biol., 2009, 19, 414-422.

3 B. Gatto, M. Palumbo and C. Sissi, Curr. Med. Chem., 2009, 16, $1248-1265$.

4 S.-H. Chou, K.-H. Chin and A. H.-J. Wang, Trends Biochem. Sci., 2005, 30, 231-234, and references therein cited; N. Jing, E. De Clercq, R. F. Randoi, L. Pallansch, C. Lackman-Smith, S. Lee and M. E. Hogan, J. Biol. Chem., 2000, 275, 3421-3430.

5 N. Jing, C. Marchand, J. Liu, R. Mitra, M. E. Hogan and Y. Pommier, J. Biol. Chem., 2000, 275, 21460-21467; J. A. Este, C. Cabrera, D. Schols, P. Cherepanov, A. Gutierrez, M. Witvrouw, C. Pannecouque, Z. Debyser, R. F. Rando, B. Clotet, J. Desmyter and E. De Clercq, Mol. Pharmacol., 1998, 53, 340-345; J. R. Wyatt, T. A. Vickers, J. L. Roberson, R. W. Buckheit, T. Klimkait, E. De Baets, P. W. Davis, B. Rayner, J. L. Imbach and D. J. Ecker, Proc. Natl. Acad. Sci. U. S. A., 1994, 91, 1356-1360.

6 H. Hotoda, M. Koizumi, R. Koga, M. Kaneko, K. Momota, T. Ohmine, H. Furukawa, T. Agatsuma, T. Nishigaki, J. Sone, S. Tsutsumi, T. Kosaka, K. Abe, S. Kimura and K. Shimada, J. Med. Chem., 1998, 41, 3655-3663.

7 J. D'Onofrio, L. Petraccone, E. Erra, L. Martino, G. Di Fabio, L. De Napoli, C. Giancola and D. Montesarchio, Bioconjugate Chem., 2007, 18, 1194-1204.

8 G. Di Fabio, C. Coppola, J. D’Onofrio, L. De Napoli and D. Montesarchio, Nucleic Acids Symp. Ser., 2008, 52, 299-300.

9 R. J. Luallen, C. Agrawal-Gamse, H. Fu, D. F. Smith, R. W. Doms and Y. Geng, Glycobiology, 2010, 20, 280-286. 